\title{
An Algorithm for Formation-Based Chemical Plume Tracing Using Robotic Marine Vehicles
}

\author{
Jorge M. Soares ${ }^{* \ddagger}$, A. Pedro Aguiar ${ }^{\dagger}$, António M. Pascoal* and Alcherio Martinoli ${ }^{\ddagger}$ \\ ${ }^{*}$ Laboratory of Robotics and Engineering Systems, Instituto Superior Técnico, University of Lisbon \\ Av. Rovisco Pais, 1049-001 Lisboa, Portugal \\ ${ }^{\dagger}$ Research Center for Systems and Technology, Faculty of Engineering, University of Porto \\ Rua Dr. Roberto Frias, 4200-465 Porto, Portugal \\ ${ }_{\ddagger}^{\ddagger}$ Distributed Intelligent Systems and Algorithms Laboratory, School of Architecture, Civil and Environmental Engineering, \\ École Polytechnique Fédérale de Lausanne (EPFL) \\ 1015 Lausanne, Switzerland
}

\begin{abstract}
Robotic chemical plume tracing is a growing area of research, with envisioned real-world applications including pollution tracking, search and rescue, and ecosystem identification. However, following a chemical signal in the water is not an easy task due to the nature of chemical transport and to limitations in sensing and communication. In this paper, we propose an approach for near-surface waterborne plume tracing using a combined team of autonomous surface and underwater vehicles. All vehicles are equipped with appropriate chemical sensors and acoustic modems. The team moves in a triangular formation, while using the flow direction and the samples obtained to steer the group along the plume. Leader vehicles at the surface implement a formation controller based on Laplacian feedback while the underwater vehicle performs acoustic ranging to the leaders. The solution was evaluated using a CFD simulation of a freshwater plume and a calibrated dynamic model of the MEDUSA autonomous marine vehicles. The group is able to move in a stable formation, sample the salinity, and trace the plume to its source.
\end{abstract}

\section{INTRODUCTION}

Following the Deepwater Horizon rig explosion [1], the resulting oil spill jetting out of the Macondo wellhead at $62000 \mathrm{barrel} /$ day [2] extended across tens of kilometers in the Gulf of Mexico, yielding one of the most recognizable images of a chemical plume. The Sentry Autonomous Underwater Vehicle (AUV), equipped with a mass spectrometer, helped map the plume [3].

Mapping oil spills or pipeline leakage is but one potential application of marine plume tracing. There are other environmental applications, such as tracing the dispersion of wastewater outfall or unauthorized pollutant discharges, or searching for hydrothermal vents, benthic structures that support complex ecosystems and release large volumes of heated water with detectable chemical signatures. As with land mines, detection of Underwater Unexploded Ordinance (UUXO) is a major concern and has traditionally been the subject of magnetic and sonar imaging methods [4], even though chemical tracing carries the potential to reduce false positives.

This work was partially funded by project FCT [UID/EEA/5009/2013] and grant SFRH/BD/51073/2010 from Fundação para a Ciência e Tecnologia.
In this paper, we look at waterborne chemical plumes and methods to track them, building upon our previous work with airborne plumes. We focus on constant-depth and near-surface operations. Although this may seem like a major limitation, water bodies are subject to stratification, with layers forming based on salinity, oxygenation, density, and temperature [5]. On short time scales, some plumes remain almost horizontal, as did the main Deepwater Horizon oil plume, at approximately $1120 \mathrm{~m}$ to $1160 \mathrm{~m}$ depth [3]. In the case of freshwater plumes in the ocean, such as those produced by river discharges, buoyancy often leads to expansion primarily along the surface [6].

To trace a near-surface plume, we propose a triangular formation of two surface vehicles and an underwater vehicle. The Autonomous Surface Vehicles (ASVs) run an adapted version of our airborne plume tracing solution built around a graph-based formation, previously described in [7], [8]. Originally conceived for terrestrial robots, this approach combines an adaptive formation-keeping component with upflow movement and plume centering based on the differences between the chemical readings obtained by each robot. The follower AUV runs a range-based formation controller [9], [10] that is able to maintain a robot in a triangular formation using acoustic ranges and piggybacked data. The underwater vehicle relays chemical concentration data, but is otherwise unaware of the plume tracing task.

We evaluate our algorithm in simulation, using the MEDUSA vehicle model and a Fluent-generated freshwater plume in a low-current saltwater medium. Using this combined approach, the vehicles are able to stay in a group, center on the plume, and scale the formation while tracing the plume to its source.

\section{RELATED WORK}

Some early work in underwater chemical source localization tried to mimic the well-studied behavior of bottom-dwelling lobsters [11], [12]. This led to the development of lobsterinspired robots [13] and Braitenberg-like controllers that link wheel speeds to the differential readings of the two antennae [14]. Other authors have looked at the active fanning behavior of crayfish [15]. 
Related bio-inspired work includes [16], [17], where the authors propose a surge-cast algorithm in which robots switch between moving at a small offset angle to up-flow and a plume reacquisition maneuver. The approach was tested in experiments with the REMUS AUV [18]. Although tangential to our work, REMUS has also been used for chemical mapping [19].

A significant body of work deals with hydrothermal vent localization. The authors of [20], [21] propose an approach in which vehicles perform a preplanned sampling survey and switch to spiral movement when a chemical signature is detected. The same team also introduced an approach based on occupancy grid maps [22], and tested their solutions using real data collected by an AUV.

In [23], the authors advance a partially-observable Markov decision process (POMDP) formulation for the hydrothermal vent localization problem and propose several planning methods building upon the aforementioned occupancy grid mapping algorithm, which are evaluated in simulation. A Bayesian inference solution using a hydrothermal plume model and artificial potential fields is proposed in [24] and also tested in a simulated environment.

An approach for cooperative 3D plume tracing using miniature robots is described in [25], based on previous 2D work with temperature plumes [26]. The authors derive a controller that allows a vehicle to independently decide its direction of motion based on a quadratic plume model and the sharing of localized concentration measurements.

Recent recognition for the field of waterborne chemical source localization comes in the form of its inclusion in the euRathlon competition [27], in both the 2014 sea edition and the 2015 grand challenge that brought together land, water and flying robots. Modeled on the Fukushima Daiichi nuclear accident of 2011 [28], relevant challenges include finding workers missing at sea and locating underwater contaminant leaks.

\section{TECHNICAL APPROACH}

Our target scenario features a neutrally buoyant surface plume, in particular a freshwater plume in a saltwater medium. The plume is detectable by sensors at the surface, as well as by sensors up to a limited depth, and is therefore traceable by a joint team of surface and underwater vehicles. The goal is to follow an already detected chemical plume to its source.

For a team entirely consisting of ASVs, the approach outlined in [8] is applicable with minor changes. However, the addition of an AUV lacking access to fully resolved relative positions precludes the exclusive use of our Laplacian feedback formation controller. We propose a set-up similar to that in [10], with two leader surface vehicles and a trailing underwater vehicle, which enables the use of our acoustic ranging approach for controlling the underwater vehicle.

We assume that the thickness of the plume does not change substantially, making it possible for the AUV to maintain a constant depth, and that an independent depth controller provides that functionality. While implementing a vertical scaling mechanism is technically straightforward [29], it requires additional data exchange and further reduces the ranging frequency, thereby impacting performance.

Our work targets the capabilities and restrictions of the MEDUSA robots [30]. We propose a controller for the surface vehicles that mirrors the one used for terrestrial robots but takes into account the different sensing and actuation modalities. In particular, the algorithm works with absolute positions and generates a control output in heading instead of angular speed. The follower runs the unmodified range-based formation controller, and all vehicles transmit their concentration readings.

The next subsections contain a description of our approach, divided into sensing, leader control, and follower control. The flexibility of our original framework ensures that minimal changes are required. Accordingly, we restate the major points of the solution but refer the reader to the previous publications for full derivations and explanations.

\section{A. Sensing and communication}

All vehicles are outfitted with appropriate chemical sensors. In our particular simulation scenario, we track a freshwater plume, discriminated by its salinity $S$. Salinity is normally measured using fast temperature-corrected conductivity probes. We can therefore assume instant salinity measurements.

Tracking a freshwater plume in saltwater requires searching for lower salinity readings, as opposed to the more common search for higher chemical concentrations. To avoid changes to the algorithm, the measured salinity can be converted to a freshness value by subtracting it from an upper bound:

$$
c_{i}=S_{\max }-S_{i}
$$

where $c_{i}$ is the resulting concentration value for vehicle $i$, $S_{i}$ is the salinity measured by its sensor, and $S_{\max }$ is a salinity upper bound appropriate to the environment, inferred from environmental measurements or known data. The mean sea water salinity is $34.7 \mathrm{ppt}$ and, in the absence of local information, $50 \mathrm{ppt}$ would be an adequate bound for the majority of surface waters. The concentrations readings are run through a $4 \mathrm{~s}$ sliding window max filter, with its output being used by the algorithm.

All vehicles have access to a magnetic compass. The underwater vehicle has no access to absolute positions, whereas the surface vehicles are assumed to be equipped with an accurate Global Positioning System (GPS) receiver. We treat the GPS positions as error-free, which is essentially the case for short-range relative positions using Real-Time Kinematic (RTK) systems. Where RTK is not available, a sensor fusion approach including GPS, Inertial Measurement Unit (IMU), and Attitude and Heading Reference System (AHRS) data should yield accurate-enough information.

We also assume at least one of the surface vehicles has some access to the direction of the current. This information may come from direct measurement, be estimated using either a Doppler Velocity Log (DVL) or GPS and a dynamical model of the vehicle, or be obtained from external sources. It may also consist solely of model data. While real-time point-accurate information may improve performance, it is not an absolute 
requirement. In strong currents, having access to the velocity of the current instead of just its direction helps improve the behavior of the vehicle.

Communication between surface vehicles is performed over short-range radios and is considered to be instant and unconstrained in data rate. This is a workable assumption given how little information is exchanged between the robots: only the most recent chemical readings and positions. We do not implement any mechanisms to cope with packet loss as the algorithm is resilient to isolated breaks in communication.

Communication with the underwater follower takes place over an acoustic link, subject to severe latency and throughput limitations. Leader vehicles send their current heading piggybacked on ranging replies when alternately queried by the follower every $2 \mathrm{~s}$. The solution departs from the pure formation-control approach in that the ranging requests sent by the follower now also include piggybacked data, namely its max-filtered concentration measurements. Both leaders listen to all requests.

\section{B. Leader control}

The two leaders implement the approach outlined in [8] with minor adjustments. The follower is part of the formation graph, but only so we can properly classify chemical readings; it is otherwise disconnected, and its formation control operates independently.

We organize our algorithm in three parallel components: up-flow movement, formation control, and plume centering. Each fulfills a particular role, but all three are required for a functional solution. The components each yield a $(\dot{x}, \dot{y})$ desired velocity vector; the three vectors are combined and transformed into control signals.

Relative positions, in the form of ranges and bearings to neighbors, are replaced with absolute positions transmitted over the radio. This is not because of a requirement of the algorithm (we showed it to work with only noisy relative positions) but because GPS is more commonly available in ASVs than relative positioning devices. This change allows us to more accurately reflect vehicle capabilities and showcases the potential for alternative implementations.

1) Laplacian feedback: We retain the generic approach from [8], only adapted to handle global positions. As we are now working with a specific triangular configuration, we propose a graph $G=(V, E)$ with

$$
\begin{aligned}
& V=\{1,2,3\} \\
& E=\{(2,3)\}
\end{aligned}
$$

and corresponding bias vectors

$$
\begin{aligned}
& \mathbf{b}_{\mathbf{u w}}=\left[\begin{array}{lll}
-1 & 0 & 0
\end{array}\right]^{T} \\
& \mathbf{b}_{\mathbf{c w}}=\left[\begin{array}{lll}
0 & -\frac{d}{2} & \frac{d}{2}
\end{array}\right]^{T}
\end{aligned}
$$

This corresponds to the formation in Figure 1, with intervehicle base distance $d$. As no edge exists connecting the trailing vehicle, its bias is not considered for formation control; any negative constant may be used.
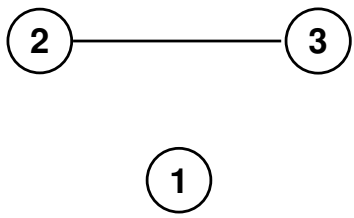

Fig. 1. Triangular marine formation showing graph edges.

Using a Laplacian feedback approach, the resulting velocity vector for formation control is given by

$$
\mathbf{u}_{\mathbf{f}}=-\left[\begin{array}{l}
\sum_{j=0}^{N} \mathcal{L}_{j}\left(x_{j}-\beta_{j}^{x}\right) \\
\sum_{j=0}^{N} \mathcal{L}_{j}\left(y_{j}-\beta_{j}^{y}\right)
\end{array}\right]
$$

where $\mathcal{L}_{j}=\mathcal{L}_{i, j}$ is the entry of the Laplacian matrix relating the controlled node $i$ to neighbor $j$, and $x_{j}$ and $y_{j}$ are the absolute positions of vehicle $j$.

The desired formation is specified in the dynamic bias $\beta_{j}$, which varies according to current and salinity readings:

$$
\beta_{j}=R(\theta)\left[\begin{array}{cc}
1 & 0 \\
0 & s_{c w}
\end{array}\right]\left[\begin{array}{l}
\mathbf{b}_{\mathbf{j}}^{\mathbf{u w}} \\
\mathbf{b}_{\mathbf{j}}^{\mathbf{c w}}
\end{array}\right]
$$

The matrix rotation $R(\theta)$ acts as a coordinate transformation between the robot and flow frames, with $\theta$ the local estimate of the current direction in the global frame. The scale in the up-flow direction is controlled solely by the follower vehicle, therefore no scaling factor is warranted. The cross-flow scaling factor $s_{c w}$ allows the formation to be scaled to adapt to the plume width, and its evolution is governed by

$$
\dot{s}_{c w}=k_{c w}\left(\left(c_{l}+c_{r}\right)-c_{c}\right)
$$

where $c_{l}, c_{r}$, and $c_{c}$ are respectively the average readings by vehicles on the left, right, and center of the formation, and $k_{c w}$ is a constant affecting the step size.

2) Up-flow movement: We implement a rheotaxis behavior by defining a movement urge in the direction of the estimated current:

$$
\mathbf{u}_{\mathbf{w}}=R(\theta)\left[\begin{array}{c}
1+\frac{|w|}{k_{w}} \\
0
\end{array}\right]
$$

In scenarios with strong currents, the base up-flow urge may not suffice to overcome the force dragging the vehicle back. If an estimate of the current speed $|w|$ is available, it can be added to the base urge. If not, the vehicles are still able to trace the plume provided the current is weaker than the urge, although the up-flow movement speed decreases accordingly.

3) Plume centering: The centering behavior assumes the formulation of a generalized logistic response on the difference between side concentrations

$$
\mathbf{u}_{\mathbf{c}}=R(\theta)\left[\begin{array}{c}
0 \\
-u_{c}^{\max }+\frac{2 u_{c}^{\max }}{1+e^{-\left(c_{l}-c_{r}\right) / k_{l}}}
\end{array}\right]
$$

where $u_{c}^{\max }$ defines the maximum absolute cross-flow adjustment and $k_{l}$ tweaks the response. 
4) Behavior aggregation: The velocity vectors for each component are combined using the weighted sum

$$
\mathbf{u}=k_{w} \mathbf{u}_{\mathbf{w}}+k_{c} \mathbf{u}_{\mathbf{c}}+k_{f} \mathbf{u}_{\mathbf{f}}
$$

As the vehicle controller takes $(v, \psi)$ inputs for navigation, the resulting desired movement vector $\mathbf{u}=\left[\begin{array}{ll}u_{x} & u_{y}\end{array}\right]^{T}$ is transformed into its magnitude and direction:

$$
\begin{aligned}
v & =\|\mathbf{u}\| & 0 \leq v \leq v_{\max } \\
\psi & =\operatorname{atan} 2\left(\mathbf{u}_{y}, \mathbf{u}_{x}\right) &
\end{aligned}
$$

\section{Follower control}

The formation controller in [10] was designed to be independent of the navigation mechanism used by the leaders. This allows us to employ the controller with no changes, keeping the follower blind to the plume tracing problem.

As the follower moves underwater and has no access to a positioning system, the controller relies on the general-purpose acoustic modem to transmit crafted echo requests. Due to the low bit rate of the medium, packets are sent alternately to each of the leaders with a minimum period of $2 \mathrm{~s}$. On receiving a request, the leaders immediately reply, and the follower can measure the round-trip time to estimate the true distance. We take advantage of the fact that ranging is performed using a data modem to piggyback the heading of each leader on the reply packets.

We skip the full derivation of the controllers and only restate the main speed and heading controller expressions, given by

$$
\begin{aligned}
& v=K_{p}^{s} \epsilon+K_{i}^{s} \int_{0}^{t} \epsilon d \tau \\
& \psi=\hat{\psi}_{f}+\gamma\left(K_{p}^{h} \delta\right)
\end{aligned}
$$

where $\epsilon=\frac{z_{2}+z_{3}}{2}-d$ and $\delta=z_{3}-z_{2}$ are the common- and differential-mode errors, derived from the ranges to the leaders $z_{1}$ and $z_{2}$ and inter-vehicle distance set-point $d, \psi_{f}$ is a Kalman estimate of the leader (formation) heading, $\gamma$ is any function such that $\sin (\gamma(a y)) y>0, \forall a>0$, and $K_{p}^{s}, K_{i}^{s}$, and $K_{p}^{h}$ are control gains.

The one difference in the follower implementation is that it now measures the salinity (more generally, chemical concentration) and relays it to the leaders as part of the acoustic ranging requests. As in the original work, we assume the follower has independent depth control and maintains a constant depth $D$.

To reduce the number of parameters, we adopt the same desired inter-vehicle distance $d$ in the leader and follower controllers, that is, for all three inter-vehicle distances. As we do not correct for the cross-flow scaling of the leader formation, and in order to ensure the stability of the controller, the scale factor $s_{c w}$ must fulfill

$$
s_{c w}<2 \sqrt{1-\left(\frac{D}{d}\right)^{2}}
$$

Larger values drive the leaders too far apart from one another and make it physically impossible for the follower to attain the desired ranges. This is not a significant limitation, as the base distance $d$ can be jointly tuned with $s_{c w}$ to achieve the desired minimum and maximum formation spans.

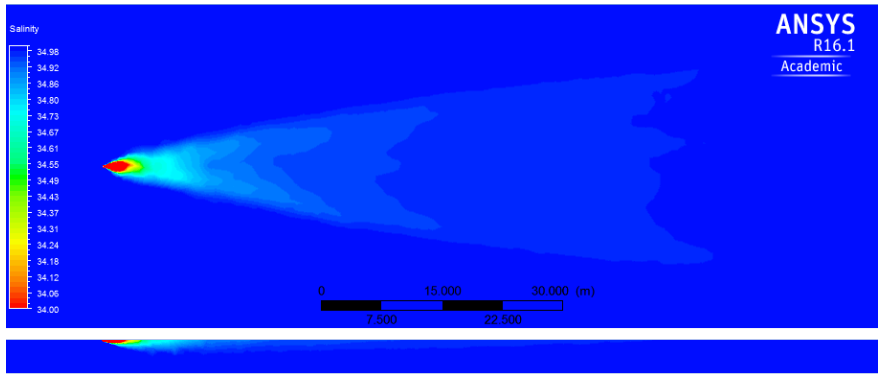

Fig. 2. Surface (top) and side (bottom) views of the freshwater plume in ANSYS Fluent. The plume measures approximately $75 \mathrm{~m} \times 25 \mathrm{~m} \times 2 \mathrm{~m}$ at $S \leq 34.98$ ppt

\section{EVALUATION}

We evaluate the complete solution in a simulated environment approaching our target scenario for future real-world trials. We run the robot simulation in MATLAB/Simulink, using virtual agents based on the MEDUSA vehicle model to trace a freshwater plume generated in ANSYS Fluent. The following sections detail the set-up and results obtained.

\section{A. Set-up}

Prior to the vehicle simulation, we generate our base plume in ANSYS Fluent. In a volume measuring $108 \mathrm{~m} \times 40 \mathrm{~m} \times 4 \mathrm{~m}$, we place a $1 \mathrm{~cm} \times 1 \mathrm{~cm}$ hose outlet at the surface, emitting freshwater in the direction of the current, at a mass flow rate of $1 \mathrm{~kg} \mathrm{~s}^{-1}$. The background current flows at $5 \mathrm{~cm} \mathrm{~s}^{-1}$ along the length of the volume.

The simulation models chemical species transport, with freshwater having salinity $0 \mathrm{ppt}$ and density $998.2 \mathrm{~kg} \mathrm{~m}^{-3}$ and saltwater having salinity $35 \mathrm{ppt}$ and density $1010 \mathrm{~kg} \mathrm{~m}^{-3}$. Both are at a default temperature of $24^{\circ} \mathrm{C}$.

We run a steady-state simulation, with standard gravity conditions and the $k-\epsilon$ viscosity model, for a total of 500 iterations, enough for the solution to converge. The plume obtained is pictured in Figure 2. Two horizontal profiles of the plume are obtained - at the surface for the ASVs, and at a depth of $0.5 \mathrm{~m}$ for the AUV - and are imported into MATLAB.

In the absence of real readings to calibrate the model, we choose to approximate the odor signatures seen in the wind tunnel and add error to the salinity readings drawn from a half-normal distribution with $\sigma=0.1 \mathrm{ppt}$, the same order of magnitude of the salinity differences between the plume and the medium. Because the freshwater plume can never cause a salinity increase, we saturate the resulting values to the medium salinity $S=35 \mathrm{ppt}$. An example snapshot is presented in Figure 3, showing the resulting patchy plume.

As we do not foresee having access to real-time current information when using the real MEDUSA vehicles, we choose to provide the robots only with the base current, $0.05 \mathrm{~m} \mathrm{~s}^{-1}$ along the length of the arena. The actual current field generating the plume is, however, non-uniform, due to the effects of the high-speed freshwater inlet and of turbulence.

The robot simulation uses the MEDUSA Simulink model for all three vehicles. The two surface vehicles run identical 

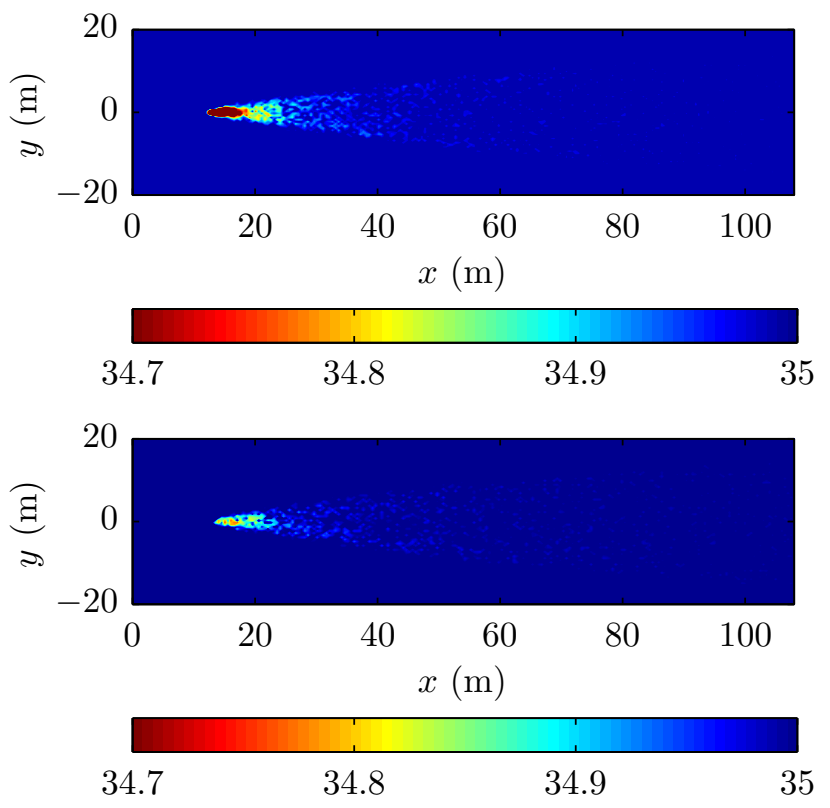

Fig. 3. Snapshot of the time-variant salinity levels with added half-normal noise, at the surface (top) and at a depth of $0.5 \mathrm{~m}$ (bottom).

copies of the leader controller whereas the underwater vehicle runs the follower controller. We only consider movement on the 2D plane, and ignore the depth of the AUV for all purposes other than chemical sampling.

The vehicles start on the right side, in the plume, and in their approximate target formation. The follower vehicle must start behind the leaders in order to converge to the desired following position.

The follower controller is configured with gains $K_{p}^{s}=0.2$, $K_{i}^{s}=0.005$, and $K_{p}^{h}=0.1$. The leader controller uses parameters $k_{c w}=5 \times 10^{-2}$ and $k_{l}=2 \times 10^{-2}$. The component weights are set to $k_{f}=0.5$ and $k_{w}=k_{c}=1$.

\section{B. Results}

We run simulations for different starting positions on the sides of the plumes. Figure 4 shows the resulting trajectories followed by the vehicles. The robots converge to the plume in all cases, although the time taken to reach the center line depends on the starting offset.

Increments to the cross-flow scaling factor are proportional to the difference in readings, which is more pronounced when exposed to the higher concentrations in the center of the plume. Therefore, the formation tends to only widen to the plume span as the robots approach its center, and formations starting closer to the plume begin to trace its limits earlier in the experiment.

Figure 5 shows the concentrations measured by each vehicle, after being put through the max filter. For the initial $250 \mathrm{~s}$ the readings increase as the vehicles make their way into and up the plume. The elevated concentrations on the right side (vehicle 3 ) cause them to move cross-flow. At $t=270 \mathrm{~s}$ the formation is centered in the plume, and the concentrations measured by the follower quickly increase. The leaders are
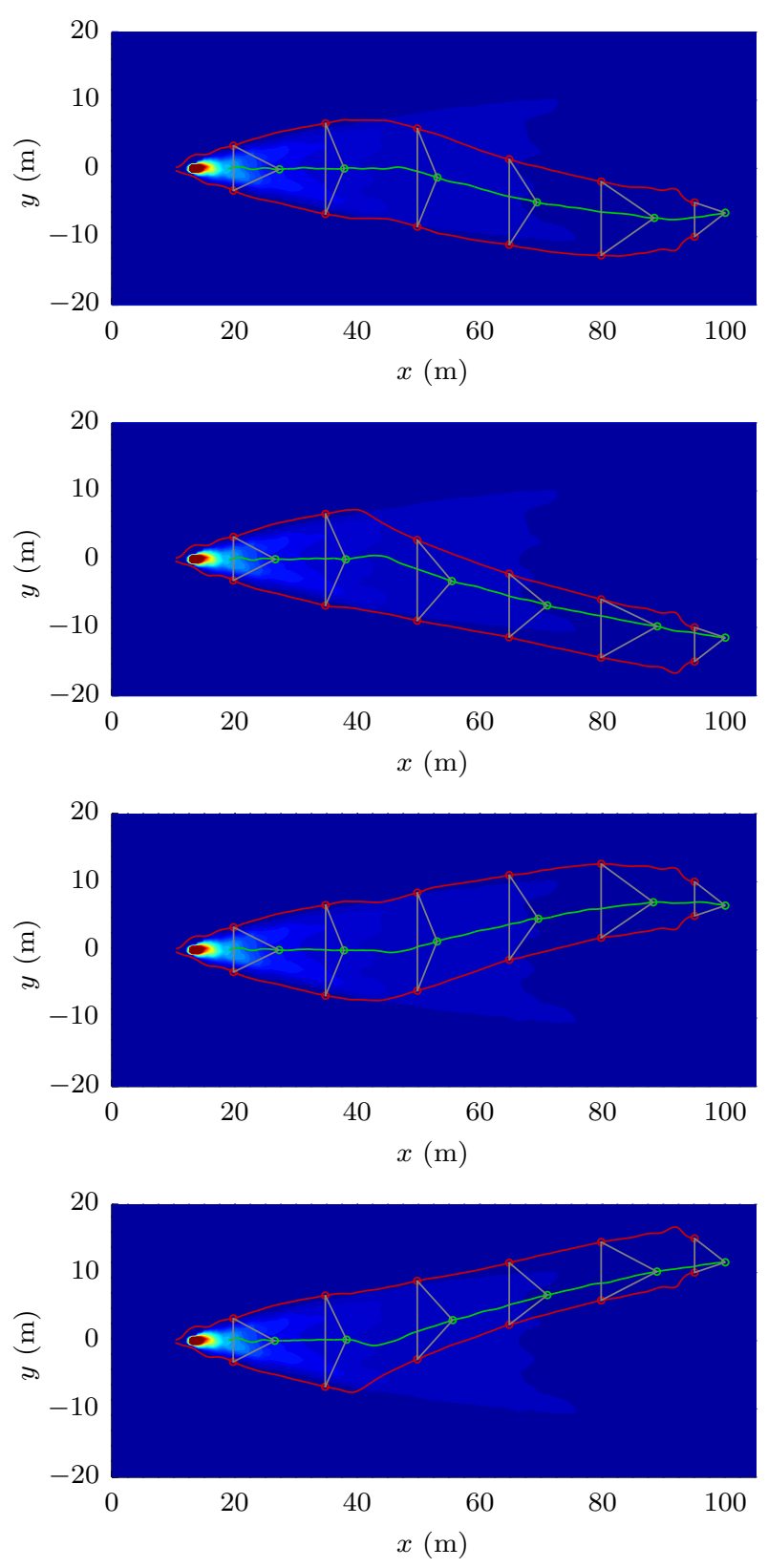

Fig. 4. Vehicle trajectories for different starting positions, superimposed on the base plume. The leader vehicles are plotted in red and the follower vehicle in green.

now on the edge of the plume and measure low concentrations, leading the formation to contract as it approaches the source.

The speed and heading of the leaders is plotted in Figure 6. Speed is mostly driven by up-flow movement and almost constant throughout the experiment, after the initial formation convergence. Whereas the up-flow heading is $270^{\circ}$, the actual heading reflects the behavior of the vehicles: in a first phase, both move at elevated headings while centering on the plume; then, their headings progress in opposite directions as the formation scales.

The follower movement, in Figure 7, is not as clean. The 


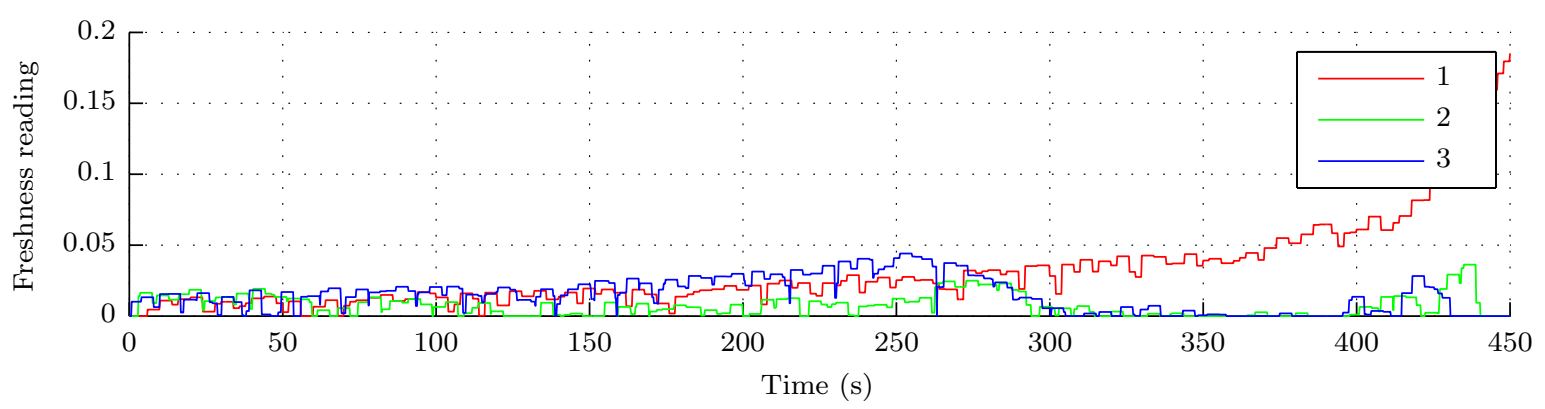

Fig. 5. Max-filtered chemical readings of all the vehicles in the first experiment in Figure 4. Vehicle 1 is the follower, and vehicles 2 and 3 are the leaders.
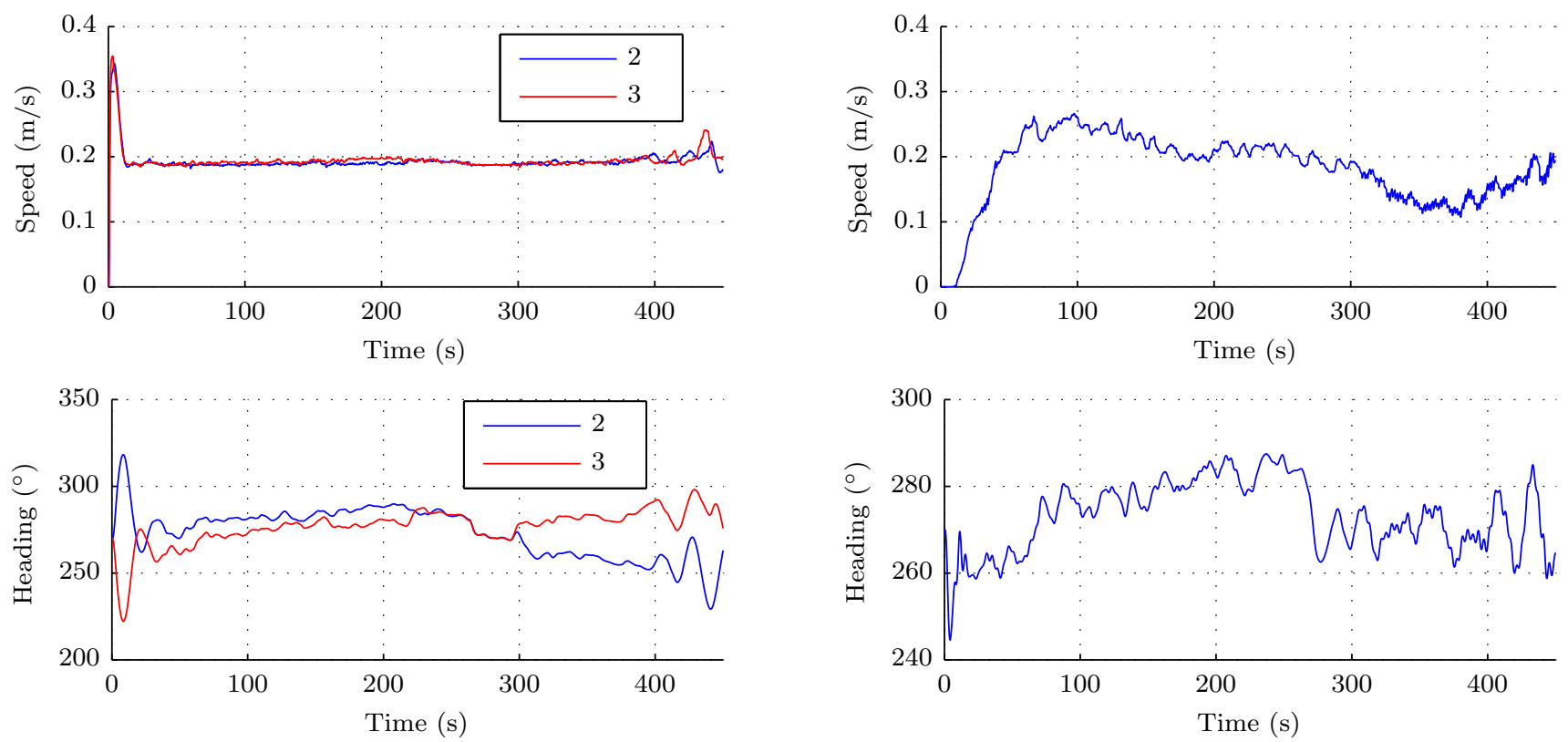

Fig. 6. Actual speed and heading of the leaders for the first experiment in Figure 4. The up-flow direction is $270^{\circ}$.

lack of accurate positioning and reliance on low-frequency range measurements introduces sawtooth disturbances in both signals, and speed is further affected by the change in interleader distance: while the leaders move at near-constant speed, formation scaling requires the follower to modulate its speed in order to move forward or back and keep its distance to the leaders. This phenomenon is most clear from $t=300 \mathrm{~s}$, when the formation narrows for the final approach to the source. The heading of the follower tracks that of the leaders during the centering phase and afterwards remains close to the up-flow direction.

\section{Alternative BehaViors}

While our solution is directly applicable to several problems, additional mechanisms may be required to handle specific scenario requirements. In the following sections, we outline two possible extensions to our methods.

Fig. 7. Actual speed and heading of the follower for the first experiment in Figure 4. The up-flow direction is $270^{\circ}$.

\section{A. Scale adjustment}

In the interest of avoiding additional data transmission, we do not feed any scale information to the follower, which always tries to achieve a preset distance to the leaders. As the leaders increase or decrease the formation span, this results in changes to the up-flow distance to the follower, i.e. the closer the leaders get to one another, the farther behind the follower lags.

Although this effect has limited impact on the performance of the solution, there are situations in which it might be desirable to compensate for it. Doing so merely requires replacing $d$ in the expressions for $\epsilon$ and $\delta$ by

$$
d_{f}=\sqrt{d_{x}^{2}+\left(s_{c w} \frac{d}{2}\right)^{2}+D^{2}}
$$

where $d_{x}$ is the desired up-flow follower offset, $d$ is the base cross-flow bias between the leaders, and $D$ is the depth of the follower. This also removes the restriction on $s_{c w}$ imposed by (16). 
Whereas all the other terms are constant and preconfigured, computing $d_{f}$ requires that the cross-flow scale coefficient $s_{c w}$ be sent to the follower over the acoustic link. Adding another numeric field to the ranging response packet, while trivial, comes at the cost of further decreased ranging frequency.

\section{B. Variable depth operation}

Assuming the follower vehicle is equipped with an appropriate depth controller, varying the depth to improve tracing performance or to gather a vertical profile of the plume can be accomplished by extending the same formation scaling approach to the vertical axis [29].

The leaders, armed with knowledge of the concentrations at the surface and underwater, can compute a scaling factor $s_{v}$ such that the follower traces the vertical shape of the plume. The follower, however, does not have access to the concentrations measured by the leaders and is unable to perform this computation.

In order to effect the depth changes, $s_{v}$ must be transmitted to the follower. Because varying the depth affects the measured ranges and further constrains the cross-flow scaling factor $s_{c w}$, this approach should be combined with that outlined in the previous subsection.

To implement the integrated solution, the leader vehicles include $\left(s_{c w}, s_{v}\right)$ in the acoustic replies sent to the follower. The follower transfers the depth reference $s_{v} D$ to the depth controller, and computes the target range $d_{f}$ using

$$
\begin{gathered}
d_{f}=\sqrt{d_{x}^{2}+\left(s_{c w} \frac{d}{2}\right)^{2}+\left(s_{v} D\right)^{2}} \\
\text { VI. CONCLUSIONS }
\end{gathered}
$$

Our method enables a triangular formation composed of two surface vehicles, acting as leaders, and an underwater vehicle, acting as follower, to trace a near-surface plume to its source.

The leaders in our combined approach implement the graphbased formation plume tracing algorithm introduced in [8]. We make only minor changes, namely the replacement of relative positions with absolute GPS coordinates, which approximate the algorithm to the sensing capabilities and constraints of ASVs while preserving its core ideas.

The follower AUV uses the range-based formation controller originally introduced in [10] to pursue the leaders, with no knowledge of the plume tracing task. In addition, it measures the concentration and relays the information as part of the ranging requests, with minimal overhead.

We demonstrated the approach working in simulation using the full dynamical model of the MEDUSA vehicles and a freshwater plume in a saltwater medium, generated using a high-fidelity Computational Fluid Dynamics (CFD) application. Despite the added complexity, the robots successfully trace the plume to its source.

A major takeaway message of this work is the little effort required to convert the land-based tracing algorithm to work with marine vehicles, and to merge properly-designed graphbased and range-based formation controllers into a hybrid approach.
An immediate next step for this research will be the implementation and evaluation of the algorithm using real vehicles. In the future, we foresee the extension of this approach to larger teams, including those with multiple underwater vehicles, and to scenarios with complex 3-D currents.

\section{REFERENCES}

[1] T. Shroder and J. Konrad, Fire on the horizon: the untold story of the gulf oil disaster. HarperCollins, 2011.

[2] M. K. McNutt, R. Camilli, T. J. Crone, G. D. Guthrie, P. A. Hsieh, T. B. Ryerson, O. Savas, and F. Shaffer, "Review of flow rate estimates of the Deepwater Horizon oil spill," Proceedings of the National Academy of Sciences of the United States of America, vol. 109, no. 50, pp. 20 260-20 267, 2012.

[3] R. Camilli, C. M. Reddy, D. R. Yoerger, B. A. S. Van Mooy, M. V Jakuba, J. C. Kinsey, C. P. McIntyre, S. P. Sylva, and J. V. Maloney, "Tracking hydrocarbon plume transport and biodegradation at Deepwater Horizon." Science, vol. 330, no. 6001, pp. 201-4, 2010.

[4] T. Clem, "Sensor technologies for hunting buried sea mines," in MTS/IEEE Oceans, vol. 1, Biloxi, MS, USA, 2002, pp. 452-460.

[5] S. A. Thorpe, An introduction to ocean turbulence. Cambridge University Press, 2007.

[6] D. A. Fong and W. R. Geyer, "The alongshore transport of freshwater in a surface-trapped river plume," Journal of Physical Oceanography, vol. 32, no. 3, pp. 957-972, 2002.

[7] J. M. Soares, A. P. Aguiar, A. M. Pascoal, and A. Martinoli, "A graph-based formation algorithm for odor plume tracing," in International Symposium on Distributed Autonomous Robotic Systems, ser. Springer Tracts in Advanced Robotics, vol. 112. Springer, 2014, pp. $255-269$.

[8] _ "A distributed formation-based odor source localization algorithm: design, implementation, and wind tunnel evaluation," in IEEE International Conference on Robotics and Automation, Seattle, WA, USA, 2015, pp. 1830-1836.

[9] J. M. Soares, A. P. Aguiar, and A. M. Pascoal, "Triangular formation control using range measurements: an application to marine robotic vehicles," in IFAC Workshop on Navigation, Guidance and Control of Underwater Vehicles, Porto, Portugal, 2012, pp. 112-117.

[10] J. M. Soares, A. P. Aguiar, A. M. Pascoal, and A. Martinoli, "Joint ASV/AUV range-based formation control: theory and experimental results," in IEEE International Conference on Robotics and Automation, Karlsruhe, Germany, 2013, pp. 5579 - 5585 .

[11] P. A. Moore, N. Scholz, and J. Atema, "Chemical orientation of lobsters, homarus americanus, in turbulent odor plumes." Journal of Chemical Ecology, vol. 17, no. 7, pp. 1293-307, 1991.

[12] J. Atema, "Eddy chemotaxis and odor landscapes: exploration of nature with animal sensors," Biological Bulletin, vol. 191, no. 1, p. 129, 1996.

[13] T. Consi, J. Atema, C. Goudey, J. Cho, and C. Chryssostomidis, "AUV guidance with chemical signals," in IEEE Symposium on Autonomous Underwater Vehicle Technology, Cambridge, MA, USA, 1994, pp. $450-455$.

[14] F. W. Grasso, T. R. Consi, D. C. Mountain, and J. Atema, "Biomimetic robot lobster performs chemo-orientation in turbulence using a pair of spatially separated sensors: Progress and challenges," Robotics and Autonomous Systems, vol. 30, no. 1-2, pp. 115-131, 2000.

[15] T. Moriizumi and H. Ishida, "Robotic systems to track chemical plumes," in Conference on Optoelectronic and Microelectronic Materials and Devices, Sydney, Australia, 2002, pp. 537-540.

[16] J. Farrell and R. Arrieta, "Biologically inspired chemical plume tracing on an autonomous underwater vehicle," in IEEE International Conference on Systems, Man and Cybernetics, vol. 7, The Hague, The Netherlands, 2004, pp. 5991-5996.

[17] J. Farrell, S. Pang, and W. Li, "Chemical plume tracing via an autonomous underwater vehicle," IEEE Journal of Oceanic Engineering, vol. 30, no. 2, pp. 428-442, 2005.

[18] J. Farrell, R. Arrieta, W. Li, and S. Pang, "Moth-inspired chemical plume tracing on an autonomous underwater vehicle," IEEE Transactions on Robotics, vol. 22, no. 2, pp. 292-307, 2006.

[19] B. Fletcher, "Chemical plume mapping with an autonomous underwater vehicle," in MTS/IEEE Oceans, vol. 1, Honolulu, HI, USA, 2001, pp. 508-512. 
[20] G. Ferri, M. V. Jakuba, and D. R. Yoerger, "A novel method for hydrothermal vents prospecting using an autonomous underwater robot," in IEEE International Conference on Robotics and Automation, Pasadena, CA, USA, 2008, pp. 1055-1060.

[21] — , "A novel trigger-based method for hydrothermal vents prospecting using an autonomous underwater robot," Autonomous Robots, vol. 29 no. 1, pp. 67-83, 2010.

[22] M. V. Jakuba and D. R. Yoerger, "Autonomous search for hydrothermal vent fields with occupancy grid maps," in Australasian Conference on Robotics and Automation, Canberra, Australia, 2008.

[23] Z. A. Saigol, "Automated planning for hydrothermal vent prospecting using AUVs," PhD Thesis, University of Birmingham, 2011.

[24] S. Pang, "Plume source localization for AUV based autonomous hydrothermal vent discovery," in MTS/IEEE Oceans, Seattle, WA, USA, 2010.

[25] R. H. Byrne, S. E. Eskridge, J. E. Hurtado, and E. L. Savage, "Algorithms and analysis for underwater vehicle plume tracing," Sandia National Laboratories," SAND2003-2643, 2003.

[26] J. Hurtado, D. Adkins, S. Eskridge, J. Harrington, E. Heller, and
R. Byrne, "Miniature mobile robots for plume tracking and source localization research," Journal of Micromechatronics, vol. 1, no. 3, pp. 253-261, 2001

[27] F. E. Schneider and D. Wildermuth, "From laboratory into real life: the EURATHLON and ELROB disaster response robotics competitions," in International Carpathian Control Conference, Szilvasvarad, Hungary, 2015, pp. 452-457.

[28] T. Ohnishi, "The disaster at Japan's Fukushima-Daiichi nuclear power plant after the March 112011 earthquake and tsunami, and the resulting spread of radioisotope contamination," Radiation Research, vol. 177, no. $1,2012$.

[29] J. M. Soares, A. Marjovi, J. Giezendanner, A. Kodiyan, A. P. Aguiar, A. M. Pascoal, and A. Martinoli, "Towards 3-D distributed odor source localization: an extended graph-based formation control algorithm for plume tracking," in IEEE/RSJ International Conference on Intelligent Robots and Systems, Daejeon, South Korea, 2016.

[30] J. Ribeiro, A. P. Aguiar, and A. M. Pascoal, "Motion control design for the Medusa robotic vehicle with experimental results," LARSyS, Lisbon, Portugal, Tech. Rep., 2012. 